\title{
SOA-Based Next Generation OSS Architecture
}

\author{
Young-Wook Woo, Daniel W. Hong, Seong-Il Kim, \\ and Byung-Soo Chang \\ Network Technology Lab., KT, \\ 463-1 Jeonmin-Dong,Yuseong-Gu,Daejeon 305-811, Korea \\ \{ywwoo,wkhong,sikim,bschang\}@kt.co.kr
}

\begin{abstract}
In convergence telecommunication environment, Business Agility plays very important role in the OSS(Operation Support System) when telco provide new merged services to customer on time. But, the OSS also becomes more and more complicated to know even what part of it should be fixed for adopting new services. This paper proposes SOA-based OSS architecture for telecommunication services in order to cope with this situation. We present the designing method of services of SOA and architecture for OSS by investigating the architectural issues of the unit of derived service elements from OSS and designing the most suitable architecture of it. By adopting the represented architecture for OSS, telco can provide new convergence service to customers faster than the competitor on the market.
\end{abstract}

Keywords: SOA, NGOSS, service, convergence, architecture

\section{Introduction}

Recently as new network technologies continue to make appearance in the market, there is a fast growth in converged products as they combine with the existing products. WiBro service will be launched as a Fixed Mobile Convergence product, while Home Networking service where various home information devices converge into a single network and IP TV that allows TV viewing through broadband Internet connection are being introduced.

In line with the launching of such convergence service products, Operation Support System (OSS) must also take on architecture where appropriate management functions can be accommodated at the right time. Still, as the OSS becomes complicated due to the addition of numerous functionalities, it often becomes more difficult to accommodate the management function. In this case, modification of even a single function in the system may cause a reverberating effect on other components, thus, the whole of chain-reaction must be taken into account upon making a modification. This process of adding or modifying a function in the OSS costs a lot of money, not to mention the difficulty in identifying the root cause of a problem when fault occurs after the launch. If legacy components have a high degree of interdependency with other components, the reusability of those existing components declines. In case of a convergence service product, due to the fact that it is a combination of several disparate services, it may be much more efficient, in terms of 
the saving of cost and time, to develop it by utilizing functionalities for the existing services. But if re-utilizing existing components is not easy, provisioning of service at the right time can become a tall task.

The most effective solution for this problem may be the Service Oriented Architecture (SOA)[1]. The basic component of SOA, service, can be loosely coupled with each other, thus weakening the relationship between components and implementing OSS by combining modularized services are possible. This way, the system can quickly respond to the addition of management functionalities for the convergence service or new functionalities for existing services. Also, as services become modularized, maintenance will become easy, and with the standardization of service interface, they will have interoperability with other modules that were developed into different platforms[2]. And, the concept of SOA can be a valuable tool for Implementing NGOSS(Next Generation Operation System and Software) program which is suggested by TM Forum.

This paper proposes next generation SOA-based OSS architecture to implement telecom services. After closely examining the architectural aspect of the service unit of the OSS and searching for the most appropriate architecture for processing them, the design methodology and OSS architecture for services, which are the basic unit in SOA configuration is proposed. In Section 2, we describe the requirements about NGOSS. And, we introduce the SOA as the OSS architecture which satisfy parts of the requirements for NGOSS. In Section 4, we suggest the method to deduce services from operations from OSS, and suggest next generation OSS architecture by describing constituent part of it. In Section 5, we will represent next generation OSS architecture is applicable to convergence service by giving an example of the WiBro service.

\section{Requirements for Next Generation OSS Architecture}

The TM Forum which is international group that provides the guidance of network operations and management present the NGOSS(New Generation Operations Systems and Software) as the guide to be the next generation OSS which has more a productive and efficient management architecture than legacy systems. It is designed to deliver measurable improvements in development and software integration environments.

There are key principles that satisfy the NGOSS architecture, such as eTOM[3], SID[4], Contract Defined Interface(CDI), Contract Registration \& Trading(CRT), Externalized Process Control(EPC) and Common Communication Vehicle(CCV)[5]. eTOM(Enhanced Telecom Operations Map) is a business framework for categorizing all the business activities that a telecommunication service provider will use. SID(Shared Information and Data model) provides an information and data reference model and common information and data vocabulary from business entity perspective. It is a logically centralized information model which guarantees data integrity and consistency in the OSS. Contract Defined Interface implies that the fundamental unit of inter- and intra-operation should be contracts which is a technology neutral representation of an interface to a service. Contract Registration \& Trading implies 
that NGOSS components can be implemented with NGOSS contracts and the components without knowledge of their physical location in the network. Externalized Process Control is about how independent business processes from the component implementation. It implies operational processes can be changed with minimum impact on the application. Common Communication Vehicle implies common communication and invocation mechanism is needed for components to communicate with each other in the OSS. These key principles of NGOSS could be implemented by the features of the architectural concept of Service Oriented Architecture

\section{Service Oriented Architecture}

SOA is a collection of services with appropriate size for users. It is software architecture for developing application completed by linking loosely coupled services as one. Service, which is the basic unit of SOA, refers to a module with a standardized interface independent of a platform. Each service is designed to be loosely coupled with other services to reduce the dependency on other modules. This allows high interoperability as well as easy modification of services. Also, the re-usability of service can be improved and cost reduced due to modularization. Creating a new service by combining existing services becomes easy, and registration and calling of these services regardless of their locations are possible by supporting location transparency. Figure 1 shows the basic Service Oriented Architecture. A service provider implements the service and publishes its description at the Service Registry. A service requester discovers and invokes the desired service registered at the Service Registry for use. Such architecture enables the service requester to search for a needed function from the Service Registry as one can shop for the most appropriate product in the market. Here, the concept of service corresponds to that of contract from NGOSS. Contract Defined Interface can be obtained by using basic architecture of SOA. Contract Registration and Trading which can be obtained by using service registry means that a defined contract shall be available for registration and trading and that location transparency shall be guaranteed.

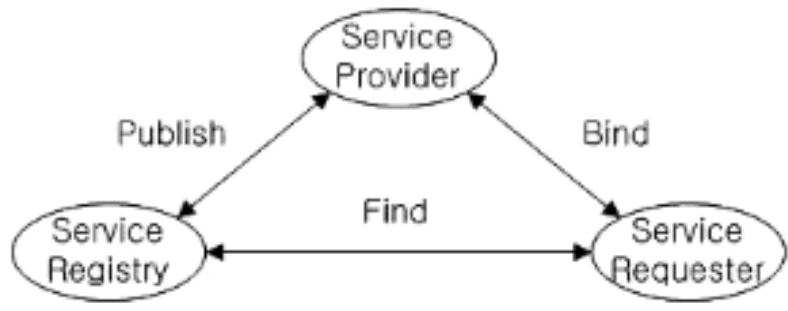

Fig. 1. Basic Service Oriented Architecture

Web service[6] is the leading technology that allows such SOA service implementation. Web service is comprised of standardized technologies independent of platform, such as Universal Description, Discovery and Integration (UDDI)[7], Web Services Description Language (WSDL)[8], and Simple Object Access Protocol 
(SOAP)[9]. Each of these has the following roles in service implementation: UDDI registers web service and provides mechanism for finding/binding which corresponds to the Service Registry in figure 1; WSDL is used to define the format of the interface and input/output message for using the web service whose role is to contain the details of services in the Service Registry of figure 1; and SOAP is the SML protocol standard that defines the message format used in web service request and response.

For the implementation of a process that is a coupling of atomic services or composite services, an integrating layer is required. For this, Business Process Management (BPM)[10], Enterprise Service Bus (ESB) and Business Activity Monitoring(BAM) are used. Figure 2 shows a system architecture that includes all of these. BPM is run as a management system and tool for process automation and management as it takes charge of the system implementation with service as the basic unit for business logic implementation. For efficient integration of distributed service components, ESB acts as a middleware that provides process-based integrated support and support for web service-based integrated environment. Aforementioned services are implemented based on consolidated database, and are coupled and orchestrated. Composite Service, which is a collection of orchestrated fundamental services, can also be used as a fundamental service for another round of orchestration. BAM plays a role of monitoring business processes which is developed by BPM and visualized the present condition of business activities of enterprise. Here, BPM and ESB can be considered as means that actually implement the concept of 'Externalized Process Control' and 'Common Communication Vehicle' of NGOSS.

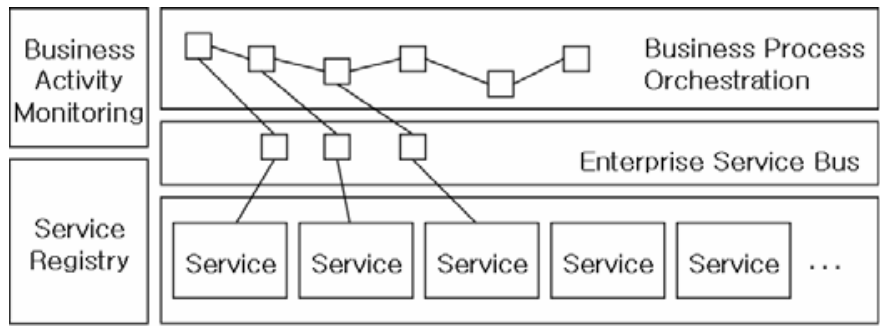

Fig. 2. Service Oriented Architecture with ESB and BPM

\section{Realization of SOA-Based Next Generation OSS Architecture}

In order to implement SOA-based Next Generation OSS Architecture, service should be defined first. And we need to define the data which will be used when services communicate each other. Before BPM orchestrate service, service management tool is required to manage services. In the following, we describe how services can be derived, a data model, service management, and orchestration in more detail. 


\subsection{Service Map}

To incorporate business logic in a system, the key issue is how to design service, the fundamental unit. Through the analysis of the functional and architectural aspects of services and the review of the performance aspect, the following criteria for decomposing services can be derived.

First, The operation map should be written by decompositioning operations for telecommunication business into hierarchical levels. For example, if Fulfillment is set as level 1 operation, below it at level 2 operation can be lower processes such as Order Entry, Resource Allocation, and Resource Configuration. Each of the Level 2 operation is divided into sub-services, and as the level goes lower, the operation unit is decomposed into the smallest possible unit. For the criteria of such decomposition, eTOM framework of NGOSS can be used as criteria in the service decomposition. And, Service map can be expanded by adding specific telco's own operations.

One of the standards of deriving services from operations may be whether a given operation has dependency on any other operation within the business process. Although an operation may complete its role by definition, there are cases where it is meaningful only as a part of the higher operation. For example, a operation called Issue Service Order does not have any significance on its own, but becomes meaningful only when a different operation in the higher service process closes the service order. Also, a operation may have a dependency on database for its execution. In other words, to run a operation, it may need to fetch data from database, rather than rely entirely on values entered at the operation calling. An example would be a operation called 'Resource Allocation.' This operation automatically allocates optimal resource to the given customer in the fulfillment process. Although customer information is provided when the operation is called, optimal resource information for customer must be searched through resource entities in database. Likewise, if a operation has a dependency on database, implementation of the operation will be affected when the database schema is modified. Therefore, an operation shall be designed to avoid such case and if it is inevitable, it shall be marked so for clear understanding.

To design and manage a system in a holistic way, the criteria of decomposition introduced above can be applied and services can be marked and managed in a table format as shown in table 1. In this paper, this is used as a service map. With such service map, specification and dependencies of operations that constitute the system can be easily discerned, which can be reflected in designing the system. It can be the tool to derive services from the operations of the system.

In the service map, decomposed operations, abstraction units of information, can be denoted by using numbers. The hierarchy of decomposed operations can be denoted in the following way: To denote a level 3 process which belongs to a level 2 process, e.g., '1.3. Resource Config.,' just adds a number to the '1.3.' to make it as '1.3.1. Fixed Resource Config.,' or '1.3.2 Mobile Resource Config.' If a operation is dependent on another, then it can be denoted by adding a prefix 'PD' (from Process Dependent service), and if independent, then 'PI' (for Process Independent service) are added to the numbers for classification. For example, an operation, '1.3.PI Resource Config.,' in table 1 shows that the operation can be used independently. When designing a system, this operation can be called by other operations even 
outside the level 1 operation Fulfillment, disregarding its relationship with other operations. If an operation is dependent on a database, a prefix 'DD' (from Database Dependent operation'), and if independent, 'DI' (from Database Independent operation) are added to the numbers respectively for classification. Operations that are independent from other operations or database are labeled 'PI.DI' and have no problem registering as service to the service registry. But if there is a dependency on either of operations or database, the registration as service to service registry will be decided according to the policy.

Table 1. An Example of the part of Service Map

\begin{tabular}{|l|l|l|l|l|}
\hline Level 1 & Level 2 & Level 3 & Level 4 & $\ldots$ \\
\hline $\begin{array}{l}\text { 1.PI.DD } \\
\text { Fulfillment }\end{array}$ & $\begin{array}{l}\text { 1.1.PD.DI } \\
\text { Order }\end{array}$ & $\begin{array}{l}\text { 1.1.1. PD.DI } \\
\text { Issue Service Order }\end{array}$ & $\cdots$ & $\cdots$ \\
\hline & & $\begin{array}{l}\text { 1.1.2.PD.DI } \\
\text { Order Receipt }\end{array}$ & $\cdots$ & $\cdots$ \\
\hline & $\begin{array}{l}\text { 1.1.3.PD.DI } \\
\text { Order Analysis }\end{array}$ & $\cdots$ & $\cdots$ \\
\hline & $\begin{array}{l}1.2 . P D . D D \\
\text { Resource Allocation }\end{array}$ & $\cdots$ & & $\cdots$ \\
& $\begin{array}{l}1.3 . P I . D I \\
\text { Resource Configuration }\end{array}$ & $\begin{array}{l}1.3 .1 . P I . D I \\
\text { Fixed Resource Configuration }\end{array}$ & $\cdots$ & $\cdots$ \\
\hline & & $\begin{array}{l}1.3 .2 . P I . D I \\
\text { Mobile Resource Configuration }\end{array}$ & $\cdots$ & $\cdots$ \\
\hline$\ldots$ & $\ldots$ & $\ldots$ & $\ldots$ & $\ldots$ \\
\hline
\end{tabular}

When an operation is decomposed as demonstrated above, the overall architecture of a system can be figured out from both business and system aspects when designing. However, operation levels may go excessively deep. If the operation level depth is excessive, the frequency of operation calling will increase, and thus make troubles regarding its performance.

After deriving the service map, services should be derived from the operations. Here, considering time delay is needed. When service is called, there exists time delay for finding the location of service from the service registry and binding the service and parsing the SOAP header. Bad network condition, lack of bandwidth also may cause time delay. Therefore, how many services can be orchestrated in the components without time performance problem should be considered when specific level of operation is decided to be service. Decomposed operations are orchestrated by BPM as shown figure 3 .

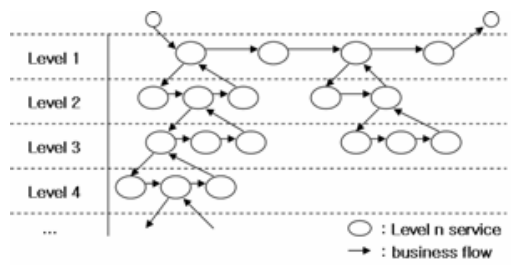

Fig. 3. Business flow of services

If service binding time ' $b$ ', represents the total time it takes when a service calls to locate another service, binds it and parses the SOAP header, and if Level n service is 
designed to call Level $n+1$ services $m$ times, the total time it takes can be calculated as follows:

$T_{\text {level }(n)}=b \times \sum_{x=1}^{m}\left(T_{\text {level }(n+1)}\right)$

If Level $n$ service is composed of a number of Level $n+1$ services, the time it takes to execute Level $n$ service will increase as the number of service decomposition levels increases. Figure 4 illustrates this in a line chart. If the number of service decomposition level is increased, the frequency of recurrent calling of sub-level services will increase, dropping the performance. Therefore, in the implementation step, it has to be decided that up to which level of decomposition shall be implemented as a service unit. The decision can be made based on the number of services, time required to call a service, and processing time of each service.

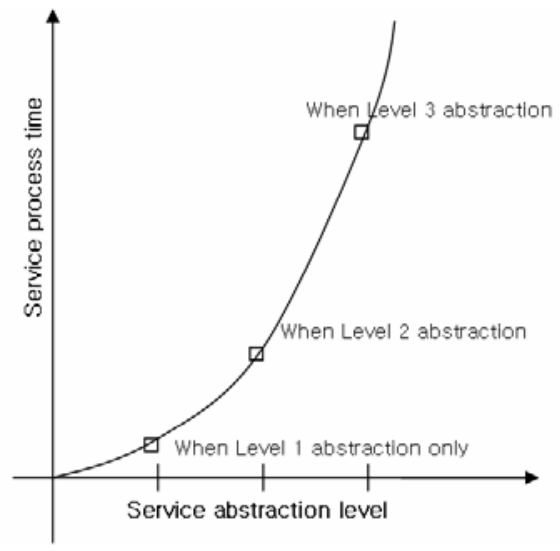

Fig. 4. Conceptual service execution time over service decomposition level

\subsection{Consolidated Database}

After every operation and service of the system are defined, the data model and repository is needed to contain the communicated data among them. The data can be modeled by analyzing operations from the service map and deriving entities and attributes from them. NGOSS suggests SID(shared Information and Data model) as data model for the common repository. It represents that whole services in the system should have shared information and data model and should not have the different data repository to deal with the same information. It can be obtained by building consolidated database, and all the services in the system should communicate each other by using the data from it. 


\subsection{Service Registry Management}

In order to manage the services derived from the service map efficiently, Service Registry Management functionalities are required. It provides the functions such as registering, updating and deleting the services to the service registry. It also provide authority management mechanism that keeps unauthorized user or system from finding and executing the important services. And, it need to keep an eye on whether the services is executed properly or not and log the result when the error occur. It also need to track and testing the execution time of each services and log the execution time of them. These logged results help system or operator making plans to use the services. And It also need to log user list and history for each services in order to prevent the services from being executed by too many systems and users at once.

\subsection{Service Orchestration}

If the functionalities to manage services are prepared, the business process can be developed by orchestration of services securely and efficiently. Figure 5 shows the SOA-based Next Generation OSS architecture which contains all the described features previously.

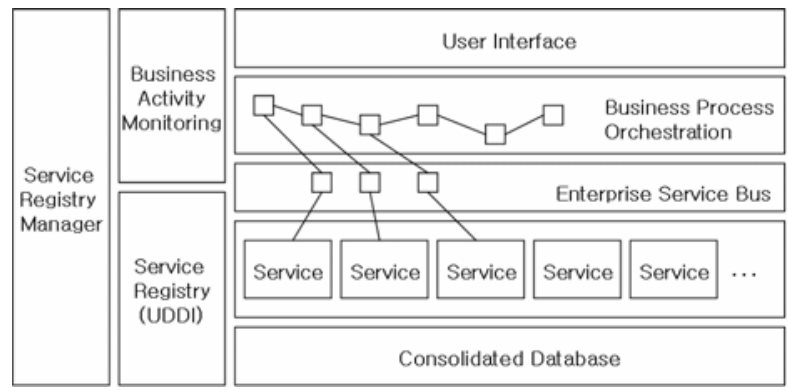

Fig. 5. SOA-based Next Generation OSS Architecture

\section{$5 \quad$ Applying SOA-Based OSS to FMC Service}

Wireless Broadband Internet (WiBro) service is a wireless mobile internet service, to be commercialized by KT in June 2005, for the first time in the world. WiBro is a leading example of FMC (Fixed Mobile Convergence) services which allows wireless access to broadband Internet. Figure 6 shows network topology of the service. 


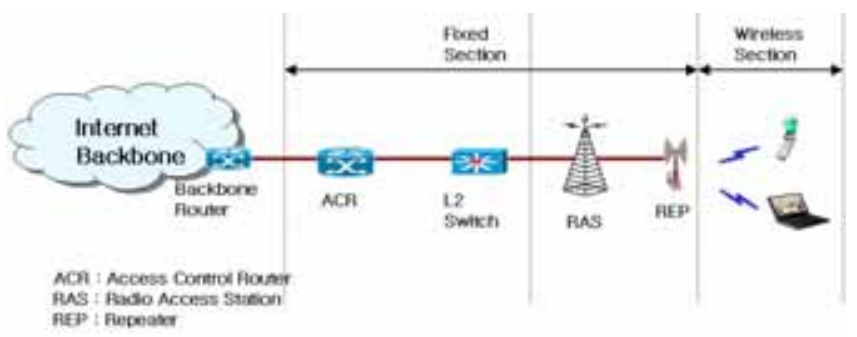

Fig. 6. WiBro Service Network Topology

WiBro requires the management of both fixed and wireless sections as shown in the network topology. When a resource trouble occurs, operators shall be able to locate in the assurance management view in which section the trouble occurred. Table 2 shows a sample of decomposed services of WiBro derived by using the aforementioned service map. Here, the service of '1.1.3.PD.DI Testing Resource Trouble' may be decomposed into two lower level services: device test functions for the fixed section and the wireless section, and then further be decomposed into test functions by equipment type in each section and services can be orchestrated and developed with the architecture which is shown in figure.5.

Table 2. The part of service map for WiBro Assurance Management

\begin{tabular}{|l|l|l|l|c|}
\hline Level 1 & Level 2 & Level 3 & Level 4 & $\ldots$ \\
\hline $\begin{array}{l}\text { 1.PI.DD } \\
\text { Assuarance }\end{array}$ & $\begin{array}{l}\text { 1.1.PD.DI } \\
\text { Resource } \\
\text { Trouble Mgmt. }\end{array}$ & $\begin{array}{l}\text { 1.1.1.PD.DI } \\
\text { Analyze Resource } \\
\text { Trouble }\end{array}$ & $\ldots$ & $\cdots$ \\
\hline & & $\begin{array}{l}1.1 .2 . P D . D I \\
\text { Create Resource } \\
\text { Trouble Report }\end{array}$ & $\cdots$ & $\cdots$ \\
\hline & $\begin{array}{l}\text { 1.1.3.PD.DI } \\
\text { Testing Resource } \\
\text { Trouble }\end{array}$ & $\begin{array}{l}\text { Testing Fixed Section Resource } \\
\text { T.1.3.PI.DI }\end{array}$ & $\ldots$ \\
\hline & & $\begin{array}{l}\text { 1.1.3.2.PI.DI } \\
\text { Testing Wireless Fixed Section } \\
\text { Resource }\end{array}$ & $\ldots$ \\
\hline$\ldots$ & $\ldots$ & $\ldots$ & $\ldots$ & $\ldots$ \\
\hline
\end{tabular}

During this process, if a testing function for the fixed section is already registered as a web service at the Service Registry, the test function module can be used during assurance management of WiBro. That is, a simple addition of a device testing function for the wireless section will be sufficient, so cost as well as development time will be reduced.

Also, by publishing the testing function as a web service, this function can be shared by other systems that also need such function. For example, as demonstrated in figure 7, it is assumed that an existing network management system in charge of Metro-Ethernet makes a device testing function for L2 switch and publishes it to the Service Registry. If WiBro service is implemented afterwards, in developing WiBro NMS, the desired Metro Switch testing function can be searched in the Service 
Registry for use. Other systems can always make use of this service from the Service Registry.

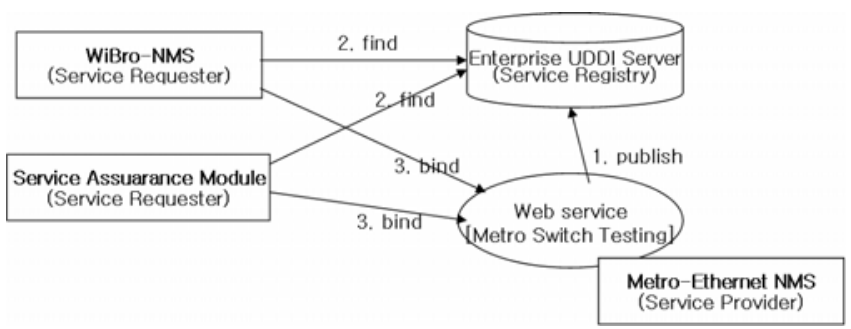

Fig. 7. An Example of Web Service [Metro Switch Testing]

\section{Conclusion}

This paper proposed SOA-based Next Generation OSS architecture which satisfies NGOSS key principles from TM Forum by applying the basic concepts of SOA. We suggested the service map which is the tool to derive services from operations and we described BPM which orchestrate services and develop business process, consolidated database which provides common communication language between each services, service registry manager which enables services can be used properly. And, this paper also presents adopting new convergence service will be easier than legacy system by reusing existing service with service repository with example of WiBro.

\section{References}

1. Roy W. Schulte Yefim V. Natis, "Service Oriented Architecture” Gartner Group, SSA Research Note SPA-401-068,1996

2. Randy Heffner, "The Big Strategic Impact Of Organic Business And Service-Oriented Architecture, Jun 2004

3. TMF, "Enhanced Telecom Operation Map (eTOM) - The Business Process Framework", GB921, V6.1 Nov 2005

4. TMF, "Shared Information and Data Model (SID), GB922, V6.1 Nov 2005

5. TMF, "The NGOSS Technology Neutral Architecture”, TMF053 (and Addenda), Aug 2004

6. W3C Web Services WG, "Web Services Architecture", http://www.w3.org/TR/2004/NOTEws-arch-20040211/, Feb 2004

7. www.uddi.org, “UDDI Technical White Paper”, Oct 2004

8. David Booth,Canyang Kevin Liu, "Web Services Description Language(WSDL) Version 2.0 Part 0: Primer”, http://www.w3.org/TR/2006/CR-wsdl20-primer-20060327, Mar 2006

9. Nilo Mitra, "SOAP Version 1.2 Part 0:Primer”, http://www.w3.org/TR/2003/REC-soap12part0-20030624/, Jun 2003

10. Howard Smith, Peter Finger, "Business Process Management the third wave", MeghanKiffer press, 2003 